\title{
Emergent Interfacial Magnetic Skyrmions and Its Technological Importance
}

\author{
Shrawan K Mishra* \\ School of Materials Science and Technology, India \\ *Corresponding author: Shrawan K Mishra, Varanasi-221005, UP, India
}

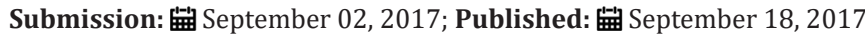

\section{Commentary}

The interface at ferromagnetic (FM) and heavy metal (HM) can display various distinct characters such as finite size effects, lattice mismatch, and broken inversion symmetries [1-5]. All these interfacial effects can provide platform for multiple emergent noncollinear spin states such as a spin helix, cycloids, and skyrmions [1-5]. Among them, nano-sized magnetic skyrmions with whirlpool like spin textures are attractive to both fundamental, as well as application-oriented research. Possible utilization of these 2D-magnetic skyrmions in future magnetic data storage will rely on the fundamental understanding of its dynamics under external stimuli like spin-polarized current pulses [1-5]. Therefore, enhanced understanding of the internal spin structure of skyrmions like size and shape is the key to predicting the interactions of skyrmions with spin currents are essential for any such promised skyrmionsbased devices [1-5].

Host materials at room temperature exhibiting skyrmionic phases are highly relevant from a technical point view. Till date, the skyrmions had been observed only in bulk metallic ferromagnetic crystal systems with broken inversion symmetry such as $\mathrm{MnSi}$, FeGe, and MnGe only at low and limited temperatures regime [1-3]. However, recently FM/HM multilayer such as $\mathrm{Pt} / \mathrm{CoFeB} / \mathrm{MgO}$ and $\mathrm{Pt} / \mathrm{Co} / \mathrm{Ir}$ have inferred from field-induced asymmetries in domain wall motion and nucleation of skyrmions at room temperature [4].

In racetrack magnetic memory device, digital information encoded as a bit in form of up and down magnetic domains separated by domain walls. Spin-polarized current drifts the bits along the bit track via the spin-transfer torque mechanism so it can travel to a magnetic domain-sensor as shown in Figure 1. The fundamental challenge that the domain walls in conventional ferromagnetic pin strongly to defects and require higher current densities to drag the domain-walls, which causes excessive Joule heating. Skyrmion can be pulled along racetrack via spin transfer torque with a very minimum current density compared to those with ferromagnetic domain walls by unique ability to deform and avoid pinning sites. Fundamental understanding of skyrmions dynamics and involved various mechanism are essential for the realization of skyrmionic based racetrack memory devices.

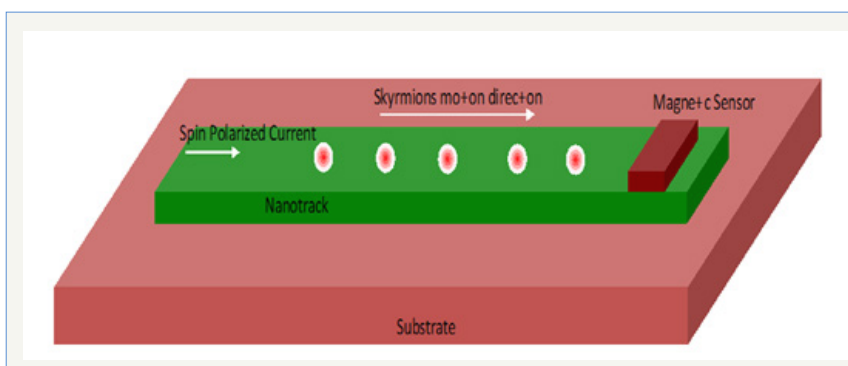

Figure 1: Skyrmions based nanotrack memory devices, where particle shaped skyrmions driven by spin torque generated from applied spin polarized currents.

The stabilized chiral domain walls such as skyrmions can lead to realizations of new nanoscale data storage by considering a memory element that stores the information by using the domainwall position [6]. Each individual domain wall can have two stable positions (magnetization) that can be measured using a magnetic tunnel junction [6]. The spin orbit torque-induced motion of chiral domain walls that relatively faster and compare to the conventional ferromagnetic domain wall is highly relevant to advancing the development of racetrack memory.

The ultimate challenge of racetrack memory is to minimize the dynamical resistance and Joule heating of devices. The governing parameters mainly can be of two major classes: electronically induced and defect induced. The electrical conductivity is precisely controlled by external voltage that provides electrical tunability that is essential for information processing technology.

In this skyrmion phase current driven collective dynamics of skyrmion will suppose to require very less current, resulting very nominal Joule heating. In presence of a strong interfacial DMI in polycrystalline thin-film stacks of $\mathrm{FM} / \mathrm{HM}$ such as $\mathrm{Pt} / \mathrm{CoFe} /$ $\mathrm{MgO}$, and $\mathrm{Pt} / \mathrm{Co} / \mathrm{Ir}$ recently have inferred from field-induced asymmetries in domain wall motion and nucleation [4] consider as an another prominent class of materials. The magnetic skyrmions and conventional magnetic domains wall in metallic ferromagnets have very similar mechanism. In racetrack memory device, digital information is encoded as a bit of up and down magnetic domains 
separated by domain walls [6]. Magnetic domains to which the information is coded are controlled and drags using current pulses. Spin polarized current pulses drift the bits along the racetrack via the spin-transfer torque mechanism so bit can travel to a magnetic domain-sensor.

To explore magnetic skyrmions for potential magnetic storage technology, we researcher need to investigate suitable conditions for the stability and dynamics of skyrmions. Due to the topologically non-trivial whirlpool-like spin arrangements and strong interaction of skyrmions with conduction electrons makes them useful for spintronic applications.

\section{References}

1. Bader SD, Parkin SSP (2010) Spintronics. Annual Review of Condensed Matter Physics 1: 71-88.
2. Rößler UK, Bogdanov AN, Pfleiderer C (2006) Spontaneous skyrmion ground states in magnetic metals. Nature 442: 797-801.

3. Mühlbauer S, Binz B, Jonietz F, Pfleiderer C, Rosch A, et al. (2009) Skyrmion Lattice in a Chiral Magnet. Science 323(5916): 915-919.

4. Olivier B, Jan V, Hongxin Y, Stefania P, Dayane DSC (2016) Roomtemperature chiral magnetic skyrmions in ultrathin magnetic nanostructures. Nature Nanotechnology 11: 449-454.

5. Stuart SPP, Masamitsu H, Thomas L (2008) Magnetic Domain-Wall Racetrack Memory. Science 320(5873): 190-194.

6. Albert F, Vincent C, João S (2013) Skyrmions on the track. Nature Nanotechnology 8: 152-156. 